\title{
Investigation of 4-hydrazinobenzoic acid derivatives for their antioxidant activity: in vitro screening and DFT study
}

\author{
Hatem A. Abuelizz ${ }^{1}$, Hanan A. A. Taie ${ }^{2}$, Ahmed H. Bakheit ${ }^{1}$, Gamal A. E. Mostafa ${ }^{1}$, \\ Mohamed Marzouk ${ }^{3}$, Harunor Rashid ${ }^{4}$, Rashad Al-Salahi ${ }^{*}$ \\ ${ }^{1}$ Department of Pharmaceutical Chemistry, College of Pharmacy, King Saud University, PO Box 2457, Riyadh \\ 11451, Saudi Arabia. Email: ralsalahi@ksu.edu.sa \\ ${ }^{2}$ Department of Plant Biochemistry, Agriculture and Biological Researches Institute, National Research Centre, \\ 33 El-Bohouth St. (Former El-Tahrir St.), Dokki, Cairo, 12622 Egypt. \\ ${ }^{3}$ Chemistry of Tanning Materials and Leather Technology Department, Chemical Industries Research Institute, \\ National Research Centre, 33 El-Bohouth St. (Former El-Tahrir St.), Dokki, Cairo, 12622, Egypt. \\ ${ }^{4}$ National Centre for Immunisation Research and Surveillance (NCIRS), Kids Research at The Children's \\ Hospital, Faculty of Medicine and Health, The University of Sydney, Westmead, NSW, 2145, Australia
}


Figure S1. Initial inputs for geometry optimizations of 1-13 and BHA

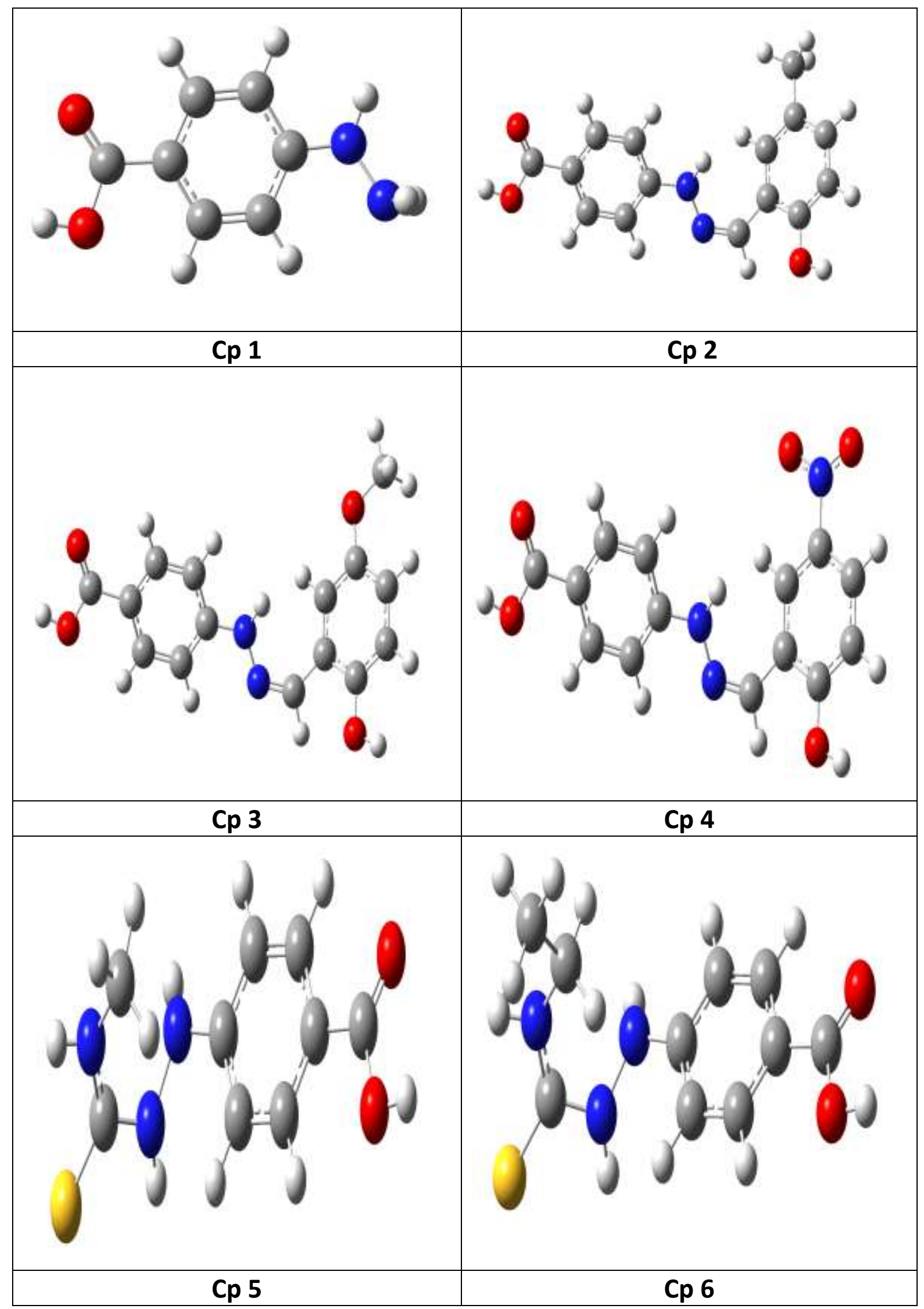




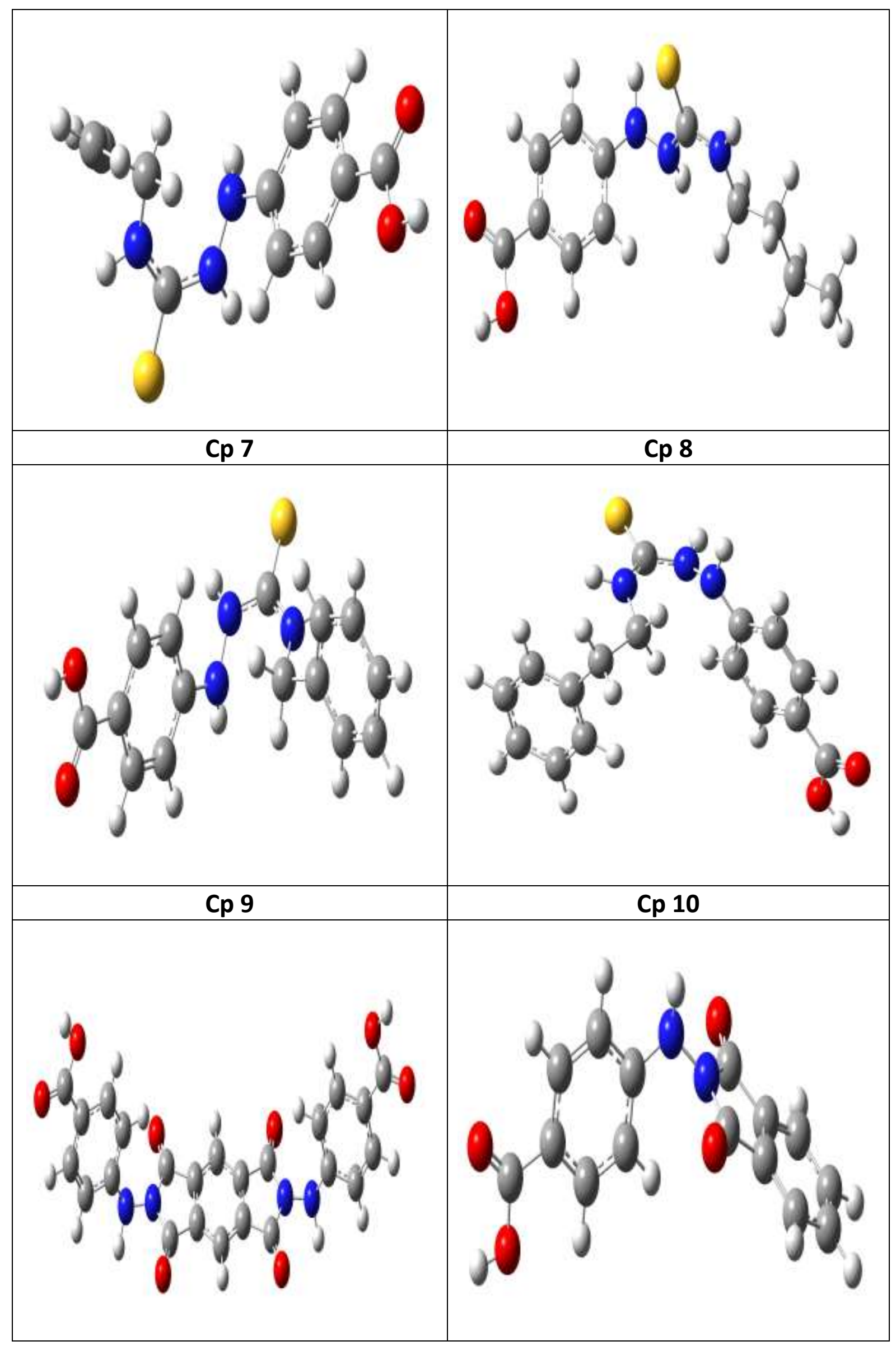




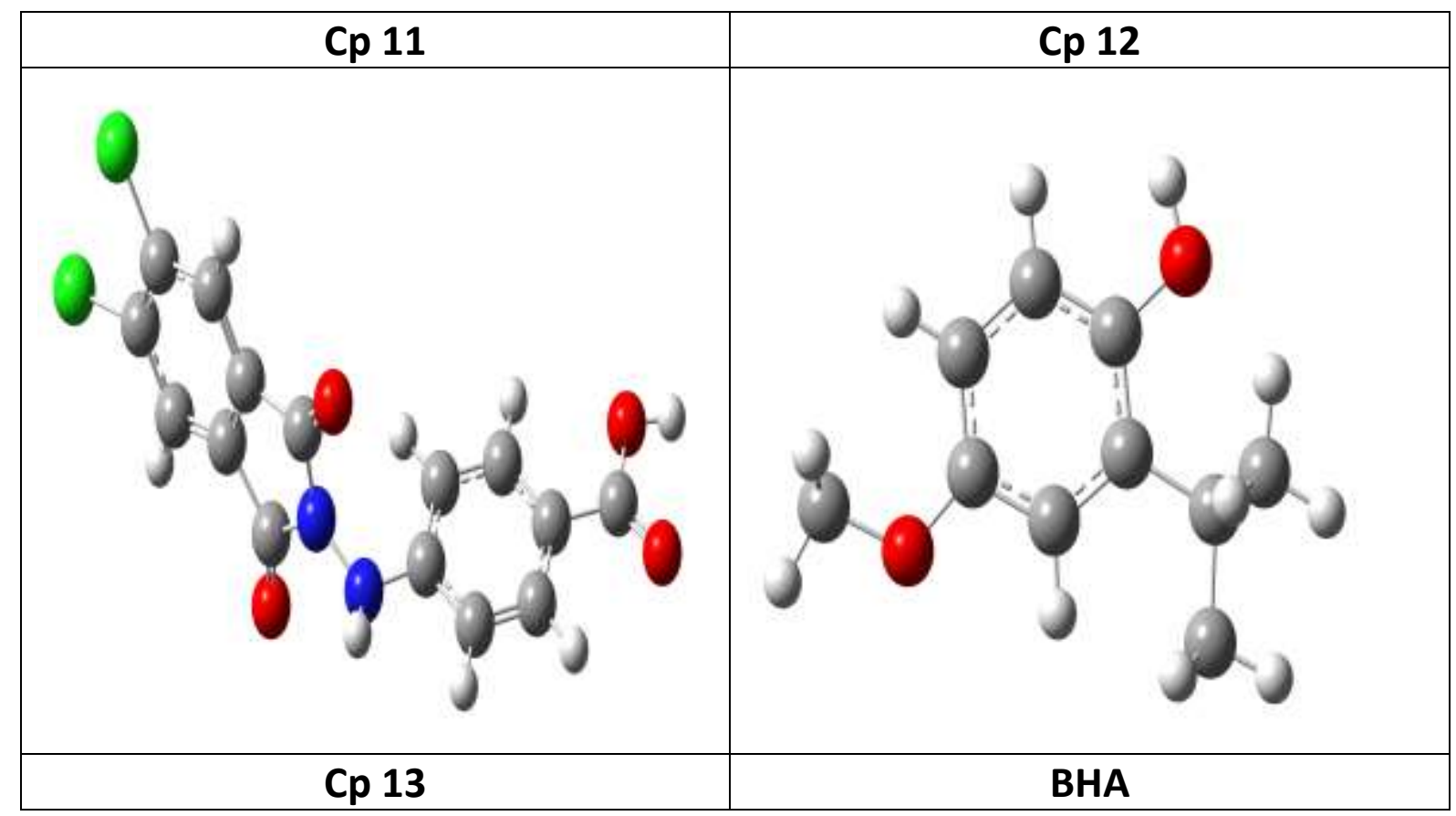

Table S1 Chemical Reactivity Descriptors

Hardness

$$
\eta=\frac{1}{2}\left(\frac{\partial^{2} E}{\partial^{2} N}\right.
$$

$\mathrm{E}$ is total energy, and $\mathrm{v}(\mathrm{r})$ is external potential. $\mathrm{N}$ is the number of electrons.

$$
\eta=\frac{1}{2}(I P-E A)
$$

IP and EA are ionization potentials and electron affinity of chemicals, respectively. 


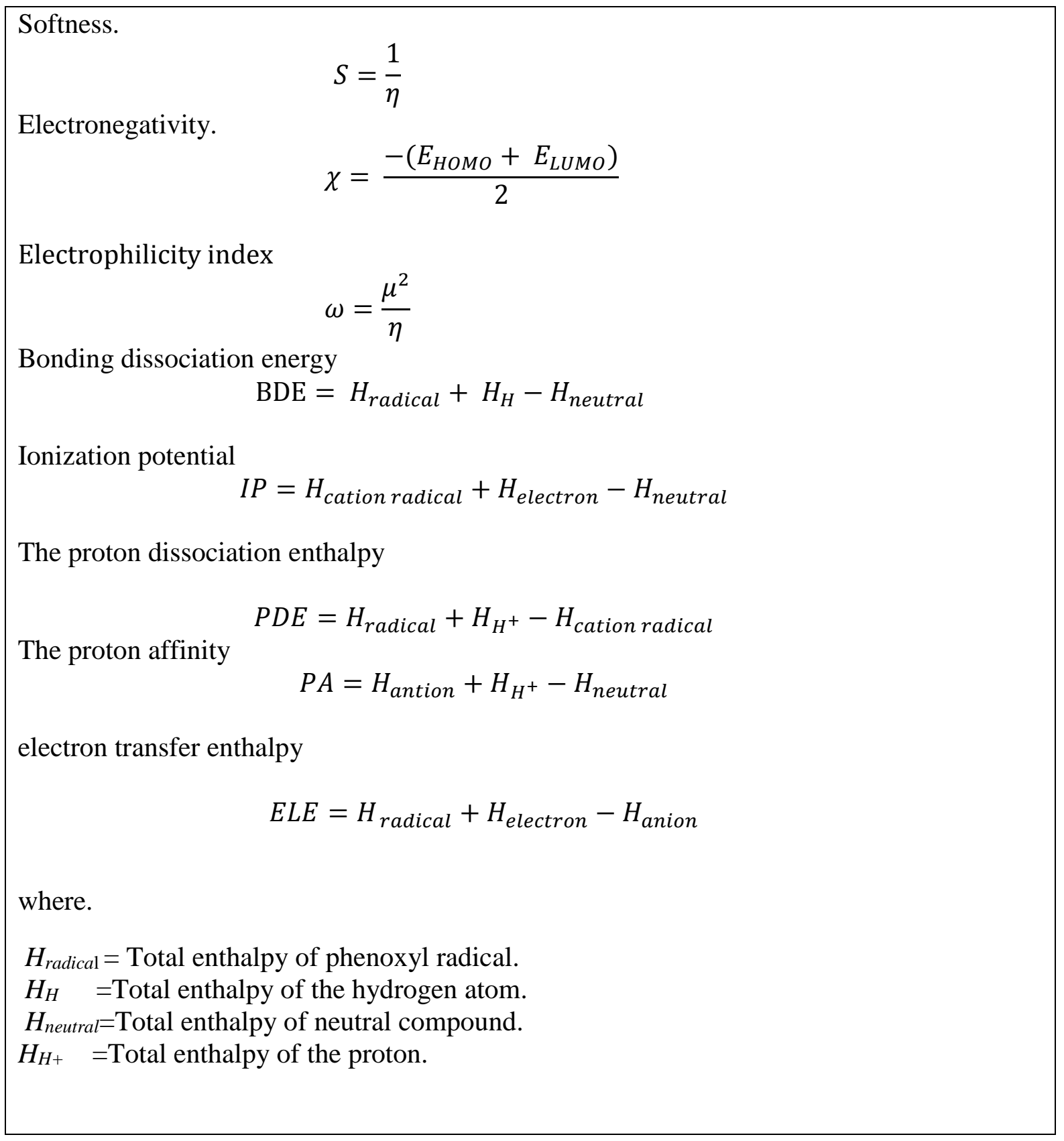


Table S2 Antioxidant reaction mechanism and computation of antioxidant descriptors

(i) Hydrogen atom transfer (HAT)

$$
\mathrm{ArXH}+\mathrm{R}^{\cdot} \rightarrow \mathrm{ArX}+\mathrm{RH}
$$

(ii) Single electron transfer (SET)

$$
\mathrm{ArXH}+\mathrm{R}^{\cdot} \rightarrow \mathrm{ArXH}^{+}+\mathrm{R}^{-} \rightarrow \mathrm{ArX}+\mathrm{RH}
$$

(iii) Sequential proton loss-electron transfer (SPLET)

$$
\begin{aligned}
& \operatorname{ArXH}+\mathrm{R}^{\cdot} \rightarrow \mathrm{ArX}^{-}+\mathrm{H}^{+} \\
& \mathrm{ArX}^{-}+\mathrm{R}^{\cdot} \rightarrow \mathrm{ArX}^{\cdot}+\mathrm{R}^{-} \\
& \mathrm{R}^{-}+\mathrm{H}^{+} \rightarrow \mathrm{RH}
\end{aligned}
$$


Scheme S1. Example of the expected reaction mechanism between DPPH free radical and compounds 5-10

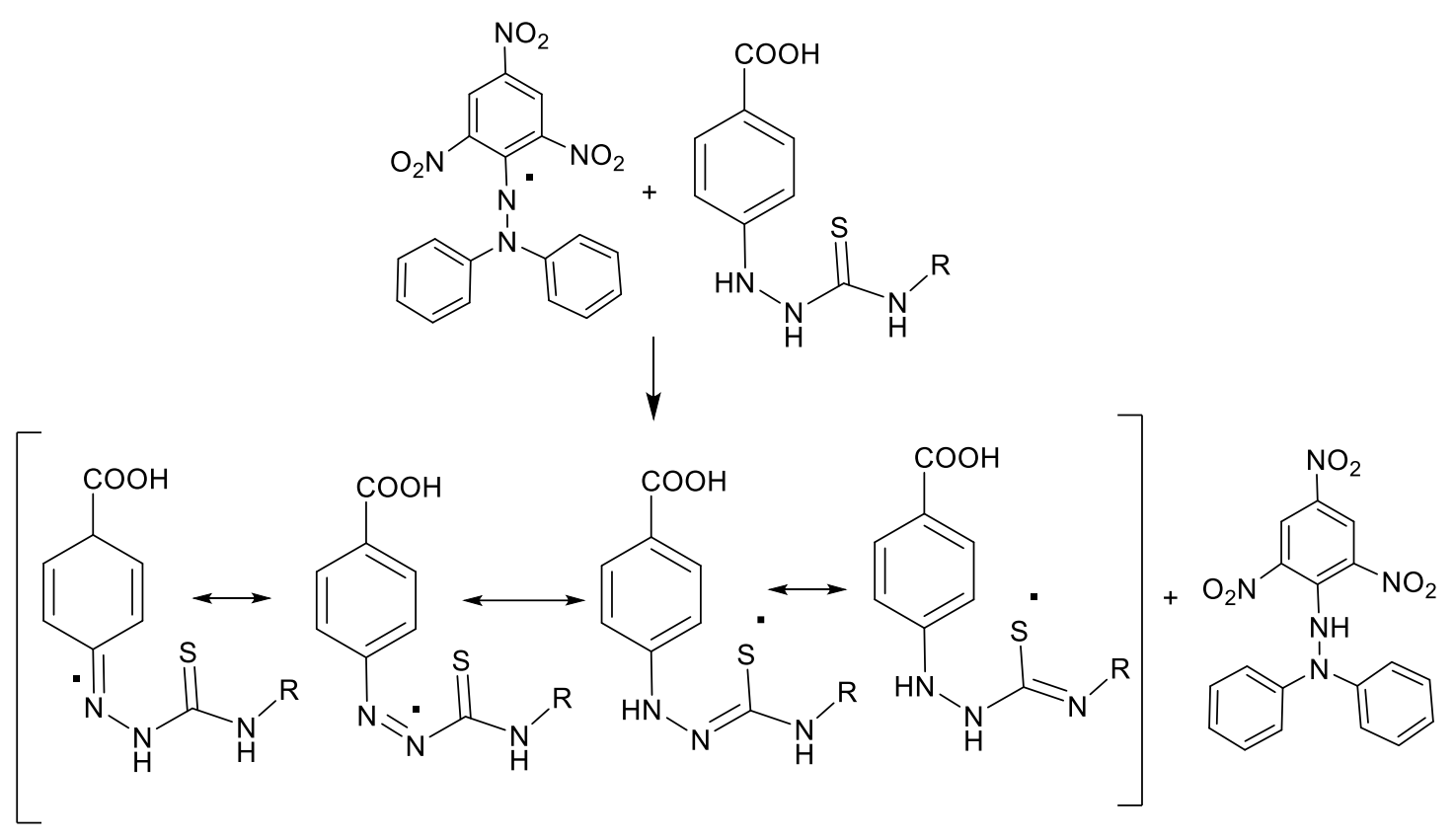

